\title{
Mini-Review
}

\section{Phenotypic switching in Cryptococcus neoformans}

\author{
Correspondence \\ B. C. Fries \\ fries@aecom.yu.edu
}

\author{
A. Guerrero, ${ }^{1}$ N. Jain, ${ }^{2}$ D. L. Goldman ${ }^{3}$ and B. C. Fries ${ }^{4}$ \\ 1,2Departments of Microbiology and Immunology ${ }^{1}$ and Department of Microbiology ${ }^{2}$ at All India \\ Institute of Medical Sciences, New Delhi, India \\ ${ }^{3,4}$ Department of Pediatrics ${ }^{3}$ and Medicine ${ }^{4}$, Albert Einstein College of Medicine, Bronx, \\ NY 10461, USA
}

\begin{abstract}
Phenotypic switching has been described in serotype A and D strains of Cryptococcus neoformans. It occurs in vivo during chronic infection and is associated with differential gene expression and changes in virulence. The switch involves changes in the polysaccharide capsule and cell wall that affect the yeast's ability to resist phagocytosis. In addition, the phenotypic switch variants elicit qualitatively different inflammatory responses in the host. In animal models of chronic cryptococosis, the immune response of the host ultimately determines which of the switch variants are selected and maintained. The importance of phenotypic switching is further underscored by several findings that are relevant in the setting of human disease. These include the ability of the mucoid colony variant of RC-2 (RC-2 MC) but not the smooth variant (RC-2 SM) to promote increased intracerebral pressure in a rat model of cryptococcal meningitis. Furthermore, chemotherapeutic and immunological antifungal interventions can promote the selection of the $\mathrm{RC}-2 \mathrm{MC}$ variant during chronic murine infection.
\end{abstract}

\section{Introduction}

Phenotypic switching enables micro-organisms to undergo rapid microevolution and to adapt to different microenvironments (Hammerschmidt et al., 1996; Lysnynansky et al., 1996; Schwan \& Hinnebush, 1998; Silverman et al., 1979; Slutsky et al., 1985). Phenotypic switching has been observed in prokaryotic and eukaryotic human pathogens, including pathogenic fungi. For human pathogens the host represents a microenvironment of particular interest and phenotypic switching in this setting can affect the hostpathogen relationship with consequences that can translate into changes in virulence. An example of how phenotypic switching can alter the host-pathogen relationship is provided by Trypanosoma cruzi, where phenotypic switching generates antigenically different variants that can escape recognition by a specific antibody (Myler et al., 1984). Other examples come from encapsulated bacteria, where phenotypic phase variation controls the expression of the capsule. This process is pivotal in determining whether the microorganism is a commensal or an invasive pathogen (Swartley et al., 1997). Because it is difficult to examine a pathogen population in vivo, studies addressing the phenotypic diversity of an isogenic population are difficult to undertake (Ebert, 1998). In this review, we will outline our findings and demonstrate why Cryptococcus neoformans represents an ideal model to examine the effects of phenotypic switching on host-pathogen interaction.

For fungi, phenotypic switching is an in vitro phenomenon defined as the spontaneous emergence of colonies with altered colony morphology at rates higher than the somatic mutation rates (Soll, 1992). Macroscopic phenotypic switching involving changes in colony morphology is associated with a diverse array of changes on the cellular level. In contrast to mass conversion, phenotypic switching occurs only in a small proportion of the pathogen population and it represents one mechanism by which 'phenotype heterogeneity' can be achieved. Phenotypic switching is reversible and thus a controlled process, which is different from a random mutation. In fungi, switching frequencies range between $10^{-2}$ and $10^{-5}$ and are higher than the expected frequency of spontaneous mutations, which in eukaryotes is between $10^{-7}$ and $10^{-8}$ mutations per generation. Phenotypic switching was first described in Candida albicans 20 years ago (Slutsky et al., 1985, 1987) and the underlying molecular mechanisms have been extensively studied (Miller \& Johnson, 2002; Perez-Martin et al., 1999). In more recent years phenotypic switching has also been demonstrated in other fungi including Cryptococcus neoformans (Eissenberg et al., 1996; Fries et al., 1999, 2001; Goldman et al., 1998; Kugler et al., 2000; Lachke et al., 2000; Sinha et al., 2000).

\section{Phenotype variability in chronic cryptococcosis}

Cryptococcus neoformans is a encapsulated yeast that causes life-threatening infections in patients with AIDS. Following the introduction of effective retroviral therapy in the United States, the annual incidence of cryptococcosis per 1000 persons with AIDS decreased approximately 10-fold, from 24-66 cases in 1992 to 2-7 cases in 2000 (Mirza et al., 2003). In less fortunate parts of the world, however, chronic 
cryptococcosis still constitutes a major opportunistic infection. In Africa, chronic C. neoformans affects up to $40 \%$ of AIDS patients (Perfect \& Casadevall, 2002). Chronic meningoencephalitis is the most common clinical manifestation of C. neoformans infection, and remains difficult to treat, despite antifungal therapy (Powderly, 2000). Lifelong suppressive antifungal therapy is recommended to reduce the likelihood of recurrent cryptococcosis in AIDS patients in whom the underlying HIV disease cannot be successfully suppressed. Treatment failures in patients with chronic cryptococcosis are generally not the result of acquired drug resistance but represent persistence of the initial strain (Brandt et al., 1996, 2001; Pfaller et al., 1999; Spitzer et al., 1993).

The propensity of $C$. neoformans to undergo rapid changes is referred to as 'microevolution'. Microevolution produces phenotypic variability that can alter the virulence of the pathogen. For the C. neoformans strain ATCC 24067 analysis of different isolates maintained in different laboratories revealed striking differences in phenotypic characteristics and virulence (Chen \& Casadevall, 1999; Franzot et al., 1998). Several observations support the concept that microevolution of $C$. neoformans occurs during the course of human infection and contributes to persistence of infection by challenging the already compromised host with variants that escape ongoing immune responses. Serial C. neoformans isolates from chronically infected HIV patients demonstrated differences in murine infection (Fries \& Casadevall, 1998) as well as changes in the polysaccharide capsule (Cherniak et al., 1995). In a similar fashion, investigations in chronic murine infection models have documented the emergence of both giant cells and poorly encapsulated forms (Feldmesser et al., 2001). In addition to the emergence of variants that exhibit changes of the sterol content in the cell membrane (Currie et al., 1995), the emergence of variants with changes in colony morphology and karyotype pattern has been described (Fries et al., 1996). Furthermore, analysis of the colony morphology in primary clinical specimens has shown that phenotypic variation in colony morphology can be observed in isolates from the spinal fluid (Fries et al., 2005a). The exact frequency of phenotypic switching in clinical isolates is not known. In a recent analysis of $C$. neoformans strains from 39 patients in India we detected phenotypic switching from a smooth to a mucoid colony morphology in 2 out of the 39 patient isolates. There is no obvious association with mating type or serotype (Jain et al., 2005). In summary, many studies demonstrate that phenotypic changes occur rapidly during chronic infection (Currie et al., 1995; Fries et al., 1996; Sukroongreung et al., 2001). Phenotypic switching is one mechanism that can facilitate microevolution.

\section{Phenotypic switching of C. neoformans strains in vitro}

For C. neoformans, phenotypic switching has so far been described in serotype A (SB4, J32) and serotype D strains (24067a, RC-2) (summarized in Table 1). In these strains colonies with altered morphology arise spontaneously at a frequency of about 1 in $10^{-4}$ to $10^{-5}$ and reversion to the parent smooth colony type occurs at a comparable frequency (approx. 1 in $10^{-3}$ to $10^{-5}$ ). The smooth colonies (S and SM) of both SB4 and 24067 are round with a smooth dome surface and smooth edges. The mucoid ( $M$ and MC) colonies are round with smooth edges and a shiny and mucoid-appearing colony surface. Wrinkled (WR), serrated (C) and pseudohyphal (PH) colonies exhibit an irregular dome surface with or without serrated margins. Both smooth and mucoid colonies represent standard colony types common to many $C$. neoformans strains, whereas the WR, C and PH colony types of SB4 and 24067a are rarely observed in

Table 1. Characteristics of C. neoformans switching strains

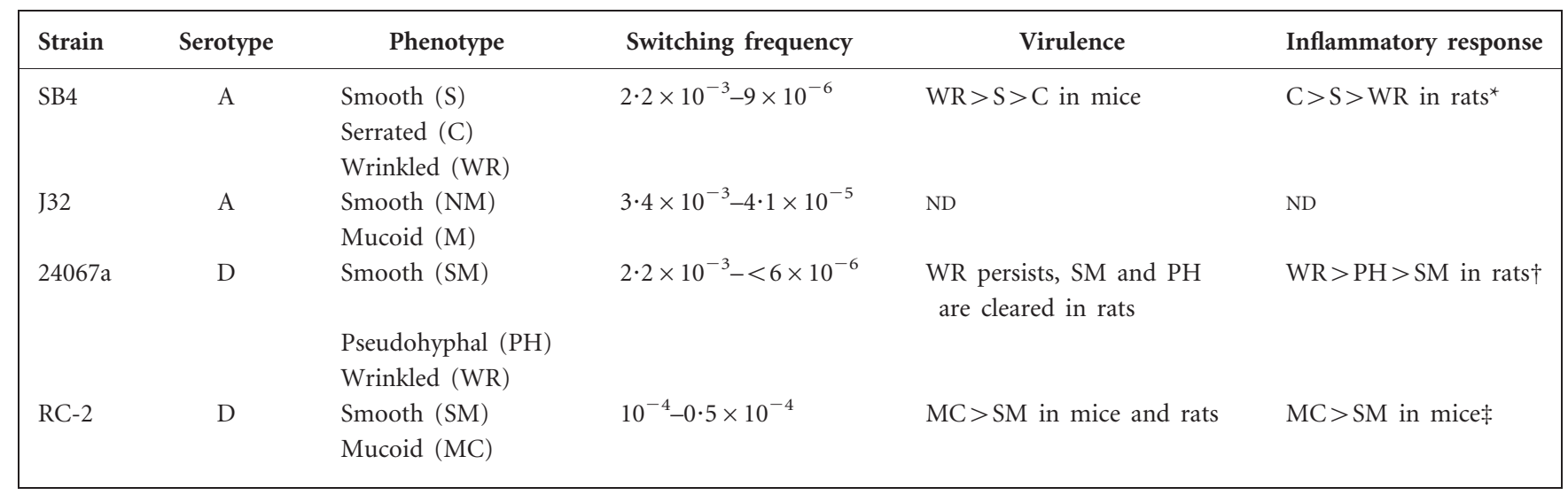

${ }^{\star}$ Minimal inflammation in WR-infected rats in contrast to intense caseous necrosis in C-infected rats.

$\dagger$ No inflammation in SM-infected rats in contrast to extensive granuloma formation in WR-infected rats.

‡Organized granuloma formation in SM-infected mice in contrast to extensive and destructive inflammatory response in MC-infected mice. 
clinical isolates. This review will focus on RC-2 because to this date it is the most thoroughly examined switching system.

The MC colonies of RC-2 exhibit an indistinguishable colony micro-architecture when compared to the parent SM colony types. This colony phenotype is the result of excessive production of a viscous exopolysaccharide which is secreted on the colony surface, leading to the shiny appearance of these colonies (Fig. 1). In contrast, the micro-architecture of $\mathrm{PH}$ colonies exhibits changes due to altered cell morphology (e.g. pseudohypal cells), and the microarchitecture of WR colonies is changed due to a change in the packing of cells. Similar changes are observed in the switching Candida strain 3153A (Radford et al., 1994).

More detailed analysis of newly generated MC switch variants demonstrates that beyond the colony morphology other phenotypic characteristics are changed (Table 2). The MC switch variant grows more slowly and exhibits a larger polysaccharide capsule compared to the SM parent strain. In older MC colonies the capsular exopolysaccharide is abundantly shed. In addition, the MC switch variant of RC-2 exhibited increased sensitivity to lysing enzyme when compared to the SM parent.

\section{Changes of the capsular polysaccharide associated with phenotypic switching}

C. neoformans is a facultative intracellular pathogen and one unique characteristic is its thick polysaccharide capsule (Bulmer \& Sans, 1968; Feldmesser et al., 2000; Levitz et al., 1999; Steenbergen et al., 2001). This capsule prevents phagocytosis and rapid destruction within the intracellular milieu of macrophages. Glucuronoxylomannan (GXM) constitutes the major exopolysaccharide of the capsule; it is composed of $(1 \rightarrow 3)$-linked linear $\alpha$-D-mannopyranan with

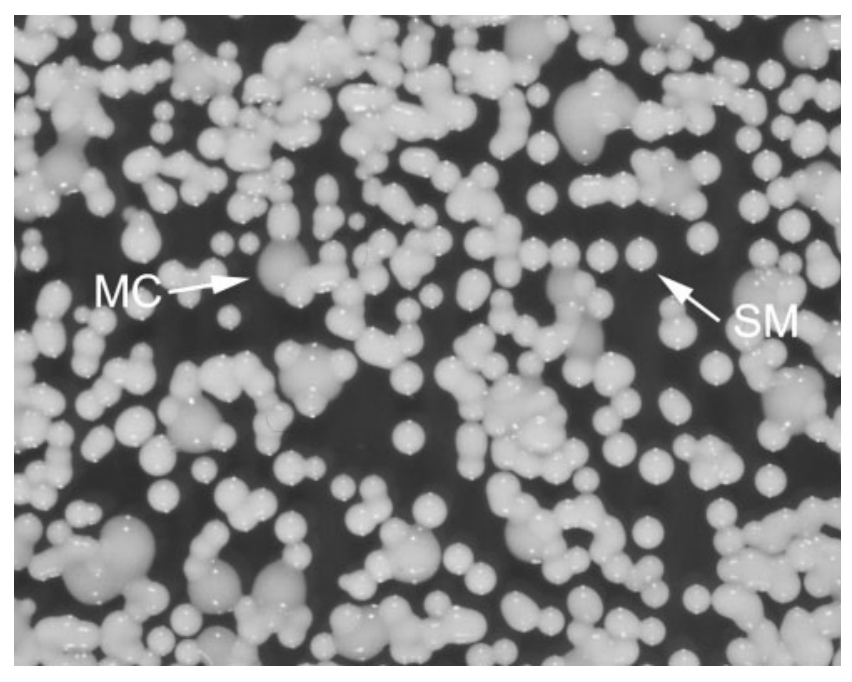

Fig. 1. SM and MC colony types of RC-2.
Table 2. Phenotypic characteristics that are affected by phenotypic switching

\begin{tabular}{|lcc|}
\hline & RC-2 SM & RC-2 MC \\
\hline Doubling time at $30{ }^{\circ} \mathrm{C}$ & $2 \cdot 5 \mathrm{~h}$ & $2 \cdot 8 \mathrm{~h}$ \\
Doubling time at $37{ }^{\circ} \mathrm{C}$ & $2 \cdot 6 \mathrm{~h}$ & $2 \cdot 7 \mathrm{~h}$ \\
Cell size at $37^{\circ} \mathrm{C}$ & $5 \cdot 9 \pm 3$ & $7 \cdot 1 \pm 0 \cdot 79$ \\
Capsule size at $37^{\circ} \mathrm{C}$ & $1 \cdot 7 \pm 0 \cdot 6$ & $2 \cdot 9 \pm 0 \cdot 54$ \\
Capsule induction in & $6 \cdot 2 \pm 09$ & $6 \cdot 9 \pm 05$ \\
$5 \% \mathrm{CO}_{2}$ & & \\
Phagocytosis index by & $39 \cdot 16 \pm 8 \cdot 6$ & $8 \cdot 9 \pm 2 \cdot 0$ \\
alveolar macrophages & & \\
Melanization & Yes & Yes \\
MIC amphotericin B & 2 & $1 \cdot 25$ \\
MIC fluconazole & $2 \cdot 5$ & $\mathrm{M} 1$ \\
GXM triad structure as & $\mathrm{M} 1$ & $\mathrm{No} \mathrm{difference}$ \\
determined by NMR & & \\
Elementary composition & No difference & $\mathrm{MC} \gg \mathrm{SM}$ \\
(C, O, H and N) & & $30 \cdot 28 \pm 3 \cdot 6 \mathrm{mV}$ \\
Viscosity of GXM & $\mathrm{MC} \gg \mathrm{SM}$ & $12 \mu \mathrm{ml}$ \\
Cell charge & $27 \cdot 68 \pm 2 \cdot 44 \mathrm{mV}$ \\
Concentration of lysing & $48 \mu \mathrm{ml} \mathrm{ml}^{-1}$ & \\
enzyme required for & & \\
complete lysis & & \\
\hline
\end{tabular}

$\beta$-D-xylopyranosyl (Xylp) and $\beta$-D-glucopyranosyluronic acid (GlcpA) residues added to the mannose at various positions. Cherniak et al. (1998) defined six structural reporter groups (SRG) based on the amount of 2-O-linked, 4-O-linked Xylp residues and 2-O-linked GlcpA residues. The type and prevalence of the GXM SRG usually correlates with differences in serological reactivity of $C$. neoformans strains. Antigenic testing has been used to classify strains according to one of five serotypes (A, D, C, B, AD).

Analogous to other encapsulated pathogens, phenotypic switching in C. neoformans can alter the exopolysaccharide. We have demonstrated that phenotypic switching results in significant changes of the biochemical composition of GXM of $\mathrm{C}$ colonies of SB4, as well as WR and PH colonies of 24067a (Fries et al., 1999). The GXMs of the C colony type are composed of mixtures of SRGs (M2 and M3 for C) whereas SB4 SM exhibits predominately SRG M2. In a similar fashion the PH and WR colonies of 24067a exhibit a mix of SRGs (M1 and M5) whereas the SM parents are predominately M1 and M2. The addition of a Xylp group at the 4-O position in M3 and M5 most likely requires a different enzyme than linkage to the $2-O$ position. Interestingly, M3 SRGs are traditionally thought to be present only in the GXM of C. neoformans var. gattii isolates (serotypes B and $\mathrm{C}$ ) and not in GXM of C. neoformans var. neoformans isolates (serotypes A and D) (Fig. 2a).

Phenotypic switching can also alter the biophysical properties of GXM (Fries et al., 2001). The MC GXM of the RC-2 strain is more viscous than the GXM of the SM parent. 
(a)

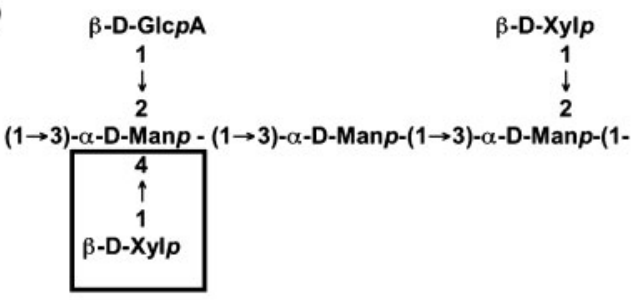

(b)

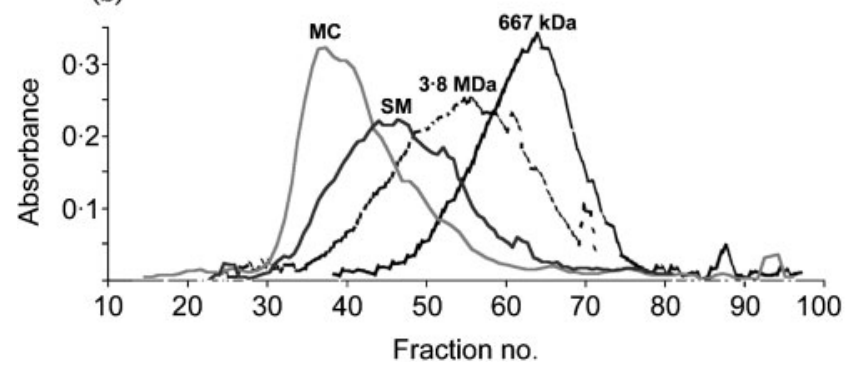

Fig. 2. GXM differences associated with phenotypic switching. (a) The core repeating triad of GXM polymer. The SRG M1, M2, M3 and M5 differ in their Xylp residue content. M3 and M5 have Xylp residues also linked in the 4-O position. (b) Size-exclusion chromatography shows earlier elution of $\mathrm{MC}$ GXM (RC-2) compared with SM GXM (RC-2), and confirms differences of the two GXMs (reproduced with permission from Fries et al. 2001).

MC GXM spins down faster during ultracentrifugation and is eluted off a size-exclusion column earlier than SM GXM (Fig. 2b). By NMR analysis, however, the SM and MC GXM exhibit the same average biochemical repeat structure (Table 2). Thus at this point it is not known what mediates the apparent difference between SM and MC GXM. Most importantly, biological assays have established that the MC GXM is more potent in inhibiting phagocytosis by macrophages in vitro and in vivo and thus contributes to the enhanced virulence of the MC switch variant in the RC-2 strain (Fries et al., 2001).

\section{Differences in virulence of phenotypic switch variants and mechanism of enhanced virulence}

In all the 'switching' $C$. neoformans strains studied so far, phenotypic switching results in variants that exhibit augmented virulence in murine or rat infection models as measured by organ fungal burden, inflammatory response and/or survival (Fig. 3). In the RC-2 switching system RC-2 MC elicits a strikingly different immune response when compared to RC-2 SM. In a pulmonary infection model in the mouse this strain elicits a more vigorous, macrophageand neutrophil-dominated inflammatory response associated with altered cytokine and chemokine expression profiles. Specifically the chemokines MCP and MIP- $1 \alpha$ were upregulated in $\mathrm{MC}$-infected mice whereas the cytokines IL-4, IL-10, IL-2 and TNF $\alpha$ were downregulated when compared to RC-2-SM-infected mice (Fries et al., 2001). In contrast, RC-2 SM elicits an effective lymphocytedominated immune response that promotes clearance of the fungal infection. The damage-driven inflammatory response in MC-infected mice leads to lung damage and rapid demise (Fig. 3). It is of note that differences in cytokine response were also observed in human peripheral blood cells that were infected with MC and SM yeast cells (Pietrella et al., 2003).

\section{Phenotypic switching of C. neoformans in vivo}

Despite an association with virulence, it is a challenge to demonstrate that switching actually occurs in the host during the infection because most experimental infections require infectious doses that are higher than the switching rate $\left(10^{-2}\right.$ to $\left.10^{-5}\right)$. As a result it is difficult to exclude that the emergence of new phenotypes in vivo is not the result of in vivo selection of altered phenotypes, derived from a mixed inoculum (Ebert, 1998). However, the characteristics of $C$. neoformans as a pathogen include two features that allowed us to prove that switching occurred in vivo: (1) the ability to induce progressive infection with a relatively small inoculum; and (2) the fact that infections are often chronic and are lethal to the host only after a prolonged period of (a)

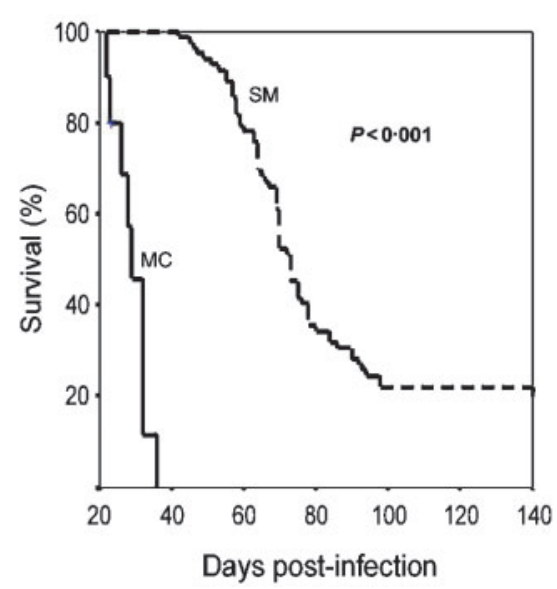

(b)

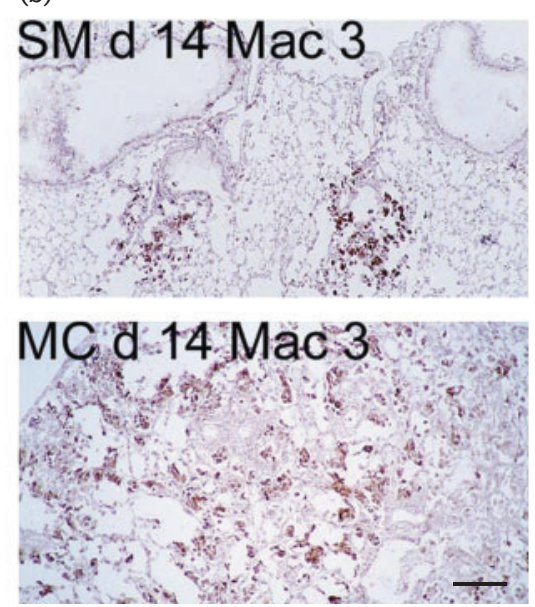

Fig. 3. (a) Mice infected with SM survive longer than mice infected intratracheally with MC (left panel). (b) Histological analysis of lung tissue with a macrophage-specific antibody (Mac-3) at day 14 shows a macrophagedominated inflammatory response in MCcompared to SM-infected lung tissue (reproduced with permission from Fries et al., 2001). Sections stained with haematoxylin and eosin; bar, $500 \mu \mathrm{m}$. 
infection. By using a small inoculum $\left(10^{3} \mathrm{SM}\right.$ cells), infecting many mice simultaneously, and applying the Poisson formula, we were able to confidently exclude the possibility $\left(P=1 \cdot 1 \times 10^{-12}\right)$ of a mixed infection as the cause for recovery of MC colony types after infection with SM (Fries et al., 2001). This result provided the first unequivocal proof that phenotypic switching occurs during the course of chronic cryptococcosis. In addition, careful analysis of primary C. neoformans isolates demonstrated that mucoid in addition to smooth colonies can be detected in primary spinal fluid specimens from humans (Fries et al., 2005b).

\section{The biological relevance of phenotypic switching during chronic infection}

The biological relevance of phenotypic switching from SM to MC in chronic infection was established in three independent studies. First, we demonstrated that the occurrence of phenotypic switching is associated with lethal outcome in murine infection. For this experiment BALB/c mice $(n=108)$ were infected with $10^{4} \mathrm{SM}$ cells, an inoculum that is generally cleared within 3 months by BALB/c mice. Mice autopsied before the onset of symptomatic disease (before day 36) and long-term survivors had significantly $(P<0 \cdot 001$, ANOVA) fewer MC colonies than those autopsied at the time of death between day 42 and day 140 (Fries et al., 2005a).

Second, we showed that antifungal treatment of the chronically infected host can promote the selection of the MC switch variants in vivo. Amphotericin B treatment reduced fungal burden less effectively in MC-infected than in SMinfected mice, and consequently resulted in a more pronounced prolongation of survival in SM-infected compared to MC-infected mice (20 versus 42 days, $P<0 \cdot 05$ ). Administration of anti-capsular monoclonal antibody mediated better protection in SM-infected than MC-infected mice, although a protective effect was not consistently observed at all doses. Most interestingly, both antifungal drug therapy and administration of anti-capsular monoclonal antibody promoted the selection of MC variants in SM-infected mice, a phenomenon manifested by a statistically higher percentage of mucoid colonies in SM-infected mice compared to non-treated control mice (Fries et al., 2005a). This finding suggests that both chemotherapeutic and immunological antifungal interventions may promote the selection of the more virulent mucoid variant, which could affect the outcome of infection in chronically infected hosts.

Third, the RC-2 MC but not the RC-2 SM variant is able to promote increased intracerebral pressure (ICP) in a rat model of cryptococcal meningitis. This finding is important because in human infection increased ICP is the leading cause of high morbidity and mortality. This complication is only seen in a subpopulation of patients, and is rather difficult to treat. Our studies suggest that $C$. neoformans strain characteristics that are altered by phenotypic switching can determine whether ICP develops during chronic infection (Fries et al., 2005b). Thus, similar to Candida (Soll et al., 1989; Soll, 2002; Vargas et al., 2000), phenotypic switching in $C$. neoformans can contribute to virulence during chronic human infection.

\section{Molecular mechanism of phenotypic switching}

The molecular mechanisms mediating phenotypic switching in C. neoformans are currently not understood. Phenotypic switching in some microbes can be achieved by genetic changes that follow a Mendelian inheritance pattern, whereas for other microbes the mechanisms are epigenetic and inherited in a non-Mendelian fashion (e.g. silencing). In Candida, white-opaque switching and sexual mating are controlled by mating type locus homeodomain proteins. In other microbes a variety of mechanisms have been implicated in generating phenotypic variability within a population, including transposition of mobile sequences, silencing of gene expression, activation of mutator genes and infection with prions, which modify the fidelity of translation termination (Chao et al., 1983; Deitsch et al., 1997; Klar et al., 2001; Lachke et al., 2000; Martin et al., 1993; Perez-Martin et al., 1999; Srikantha et al., 2001; True \& Lindquist, 2000).

Although karyotype instability was observed in strains 24067a and SB4 (Fries et al., 1999; Goldman et al., 1998), similar to the switching C. albicans strain 3153A, it could not consistently be correlated with phenotypic variability and was not reversible (Perez-Martin et al., 1999; RustchenkoBulgac, 1991; Soll, 1992). Investigations involving differential display of RC-2 SM and RC-2 MC mRNA demonstrated that phenotypic switching was associated with downregulation of genes in the MC switch variant relative to the SM switch variant. The function of the majority of these genes is unknown; however, some may represent immunogenic epitopes (Guerrero et al., 2003). Future studies will be directed at determining the specific function of the individual genes.

\section{In conclusion}

The phenomenon of phenotypic switching has been described for many different types of pathogens, and it enables pathogens to adapt to a changing environment. Such environments include different hosts that will employ sophisticated immunological tools to rid themselves of the pathogen, and variable environmental niches where microbes can be subjected to many types of selection pressures. Virulence of a pathogen is dependent on the pathogen's interaction with the host; thus it may not be a fixed characteristic of the pathogen but the product of a complex interaction between the host and the pathogen (Casadevall \& Pirofski, 1999, 2001). Changes of the host's immune competence as well as changes of the pathogen's virulence factors affect this complex relationship.

C. neoformans is an excellent model organism to study phenotypic switching and its impact on progression of chronic infection. Data from our laboratory demonstrated for the first time phenotypic switching in a fungus during 
experimental infection (Fries et al., 2001). The importance of this finding is underscored by the demonstration that the phenotypic switch changes the outcome of infection (D'Souza \& Heitman, 2001) and that selection of switch variants is promoted in the setting of antifungal therapy. C. neoformans is a haploid fungus that exhibits an asexual reproduction mode during human infection. Molecular studies suggest that $C$. neoformans populations have a largely clonal population structure that may have limited options for rapid change in response to environmental stress. Similarly, the limited virulence traits of $C$. neoformans in the setting of chronic infection may require constant microevolution to evade the immune response. We propose that phenotypic switching allows this pathogen rapid change without the disadvantage of an increased mutation rate, thus avoiding the accumulation of disadvantageous mutations. Future studies will concentrate on elucidating the underlying mechanism for phenotypic switching, which may differ for individual strains.

\section{Acknowledgements}

This work was supported by R0-1 AI 59681 to B.C.F; N.J. was supported by the AIDS International Training and Research Program (NID D43-TW01403) of the Albert Einstein College of Medicine.

\section{References}

Brandt, M. E., Pfaller, M. A., Hajjeh, R. A., Graviss, E. A., Rees, J., Spitzer, E. D., Pinner, R. W. \& Mayer, L. W. (1996). Molecular subtypes and antifungal susceptibilities of serial Cryptococcus neoformans isolates in human immunodeficiency virus-associated cryptococcosis. Cryptococcal Disease Active Surveillance Group. J Infect Dis 174, 812-820.

Brandt, M. E., Pfaller, M. A., Hajjeh, R. A., Hamill, R. J., Pappas, P. G., Reingold, A. L., Rimland, D. \& Warnock, D. W. (2001). Trends in antifungal drug susceptibility of Cryptococcus neoformans isolates in the United States: 1992 to 1994 and 1996 to 1998. Antimicrob Agents Chemother 45, 3065-3069.

Bulmer, G. S. \& Sans, M. D. (1968). Cryptococcus neoformans. III. Inhibition of phagocytosis. J Bacteriol 95, 5-8.

Casadevall, A. \& Pirofski, L. A. (1999). Host-pathogen interactions: redefining the basic concepts of virulence and pathogenicity. Infect Immun 67, 3703-3713.

Casadevall, A. \& Pirofski, L. (2001). Host-pathogen interactions: the attributes of virulence. J Infect Dis 184, 337-344.

Chao, L., Vargas, C., Spear, B. \& Cox, E. (1983). Transposable elements as mutator genes in evolution. Nature 303, 633-635.

Chen, L. C. \& Casadevall, A. (1999). Variants of a Cryptococcus neoformans strain elicit different inflammatory responses in mice. Clin Diagn Lab Immunol 6, 266-268.

Cherniak, R., Morris, L. C., Belay, T., Spitzer, E. D. \& Casadevall, A. (1995). Variation in the structure of glucuronoxylomannan in isolates from patients with recurrent cryptococcal meningitis. Infect Immun 63, 1899-1905.

Cherniak, R., Valafar, H., Morris, L. \& Valafar, F. (1998). Cryptococcus neoformans chemotyping by quantitative analysis of ${ }^{1} \mathrm{H}$ nuclear magnetic resonance spectra of glucuronoxylomannans with a computer-simulated artificial neural network. Clin Diagn Lab Immunol 5, 146-159.
Currie, B., Sanati, H., Ibrahim, A. S., Edwards, J. E., Jr, Casadevall, A. \& Ghannoum, M. A. (1995). Sterol compositions and susceptibilities to amphotericin B of environmental Cryptococcus neoformans isolates are changed by murine passage. Antimicrob Agents Chemother 39, 1934-1937.

Deitsch, K. W., Moxon, E. R. \& Wellems, T. E. (1997). Shared themes of antigenic variation and virulence in bacterial, protozoal, and fungal infections. Microbiol Mol Biol Rev 61, 281-293.

D'Souza, C. A. \& Heitman, J. (2001). It infects me, it infects me not: phenotypic switching in the fungal pathogen Cryptococcus neoformans. J Clin Invest 108, 1577-1578.

Ebert, D. (1998). Experimental evolution of parasites. Science 282, 1432-1435.

Eissenberg, L. G., Poirier, S. \& Goldman, W. E. (1996). Phenotypic variation and persistence of Histoplasma capsulatum yeasts in host cells. Infect Immun 64, 5310-5314.

Feldmesser, M., Kress, Y., Novikoff, P. \& Casadevall, A. (2000), Cryptococcus neoformans is a facultative intracellular pathogen in murine pulmonary infection. Infect Immun 68, 4225-4237.

Feldmesser, M., Kress, Y. \& Casadevall, A. (2001). Dynamic changes in the morphology of Cryptococcus neoformans during murine pulmonary infection. Microbiology 147, 2355-2365.

Franzot, S., Mukherjee, J., Cherniak, R., Chen, L., Hamdan, J. \& Casadevall, A. (1998). Microevolution of a standard strain of Cryptococcus neoformans resulting in differences in virulence and other phenotypes. Infect Immun 66, 89-97.

Fries, B. C. \& Casadevall, A. (1998). Serial isolates of Cryptococcus neoformans from patients with AIDS differ in virulence for mice. J Infect Dis 178, 1761-1766.

Fries, B. C., Chen, F., Currie, B. P. \& Casadevall, A. (1996). Karyotype instability in Cryptococcus neoformans infection. J Clin Microbiol 34, 1531-1534.

Fries, B. C., Goldman, D. L., Cherniak, R., Ju, R. \& Casadevall, A. (1999). Phenotypic switching in Cryptococcus neoformans results in changes in cellular morphology and glucuronoxylomannan structure. Infect Immun 67, 6076-6083.

Fries, B. C., Taborda, C. P., Serfass, E. \& Casadevall, A. (2001). Phenotypic switching of Cryptococcus neoformans occurs in vivo and influences the outcome of infection. J Clin Invest 108, 1639-1648.

Fries, B. C., Cook, E., Wang, X. \& Casadevall, A. (2005a). Effects of antifungal interventions on the outcome of experimental infections with phenotypic switch variants of Cryptococcus neoformans. Antimicrob Agents Chemother 49, 350-357.

Fries, B. C., Lee, S. C., Kennan, R., Zhao, W., Casadevall, A. \& Goldman, D. L. (2005b). Phenotypic switching of Cryptococcus neoformans can produce variants that elicit increased intracranial pressure in a rat model of cryptococcal meningoencephalitis. Infect Immun 73, 1779-1787.

Goldman, D., Fries, B., Franzot, S., Montella, L. \& Casadevall, A. (1998). Phenotypic switching in the human pathogenic fungus Cryptococcus neoformans is associated with changes in virulence and pulmonary inflammatory response in rodents. Proc Natl Acad Sci U S A 95, 14967-14972.

Guerrero, A., Jain, N., Cook, E., Casadevall, A. \& Fries, B. (2003). Phenotypic switch variants of Cryptococcus neoformans differ in gene expression profile. In 103rd General Meeting of the American Society for Microbiology, Washington DC, Abstract F39.

Hammerschmidt, S., Hilse, R., van Putten, J., Gerady-Schahn, R., Unkmair, A. \& Frosch, M. (1996). Modulation of cell surface sialic acid expression in Neisseria meningitidis via a transposable genetic element. EMBO J 15, 192-198. 
Jain, N., Wickes, B. L., Keller, S. M., Fu, J., Casadevall, A., Jain, P., Ragan, M. A., Banerjee, U. \& Fries, B. J Clin Microbiol 43, 5733-5742.

Klar, A. J., Srikantha, T. \& Soll, D. R. (2001). A histone deacetylation inhibitor and mutant promote colony-type switching of the human pathogen Candida albicans. Genetics 158, 919-924.

Kugler, S., Schurtz Sebghati, T., Groppe Eissenberg, L. \& Goldman, W. E. (2000). Phenotypic variation and intracellular parasitism by Histoplasma capsulatum. Proc Natl Acad Sci U S A 97, 8794-8798.

Lachke, S. A., Srikantha, T., Tsai, L. K., Daniels, K. \& Soll, D. R. (2000). Phenotypic switching in Candida glabrata involves phasespecific regulation of the metallothionein gene MT-II and the newly discovered hemolysin gene HLP. Infect Immun 68, 884-895.

Levitz, S. M., Nong, S. H., Seetoo, K. F., Harrison, T. S., Speizer, R. A. \& Simons, E. R. (1999). Cryptococcus neoformans resides in an acidic phagolysosome of human macrophages. Infect Immun 67, 885-890.

Lysnynansky, I., Rosengarten, R. \& Yogev, D. (1996). Phenotypic switching of variable surface lipoproteins in Mycoplasma bovis involves high-frequency chromosomal rearrangement. J Bacteriol 178, 5395-5401.

Martin, D. W., Schurr, M. J., Mudd, M. H., Govan, J. R., Holloway, B. W. \& Deretic, V. (1993). Mechanism of conversion to mucoidy in Pseudomonas aeruginosa infecting cystic fibrosis patients. Proc Natl Acad Sci U S A 90, 8377-8381.

Miller, M. G. \& Johnson, A. D. (2002). White-opaque switching in Candida albicans is controlled by mating-type locus homeodomain proteins and allows efficient mating. Cell 110, 293-302.

Mirza, S. A., Phelan, M., Rimland, D. \& 8 other authors (2003). The changing epidemiology of cryptococcosis: an update from population-based active surveillance in 2 large metropolitan areas, 1992-2000. Clin Infect Dis 36, 789-794.

Myler, P., Allison, J., Agabian, N. \& Stuart, K. (1984). Antigenic variation in African trypanosomes by gene replacement or activation of alternate telomeres. Cell 39, 203-211.

Perez-Martin, J., Uria, J. A. \& Johnson, A. D. (1999). Phenotypic switching in Candida albicans is controlled by a SIR2 gene. EMBO J 18, 2580-2592.

Perfect, J. R. \& Casadevall, A. (2002). Cryptococcosis. Infect Dis Clin North Am 16, 837-874, v-vi.

Pfaller, M. A., Zhang, J., Messer, S. A., Brandt, M. E., Hajjeh, R. A., Jessup, C. J., Tumberland, M., Mbidde, E. K. \& Ghannoum, M. A. (1999). In vitro activities of voriconazole, fluconazole, and itraconazole against 566 clinical isolates of Cryptococcus neoformans from the United States and Africa. Antimicrob Agents Chemother 43, 169-171.

Pietrella, D., Fries, B., Lupo, P., Bistoni, F., Casadevall, A. \& Vecchiarelli, A. (2003). Phenotypic switching of Cryptococcus neoformans can influence the outcome of the human immune response. Cell Microbiol 5, 513-522.

Powderly, W. G. (2000). Current approach to the acute management of cryptococcal infections. J Infect 41, 18-22.

Radford, D., Challacombe, S. \& Walter, J. (1994). A scanning electron microscopy investigation of the structure of colonies of different morphologies produced by phenotypic switching in Candida albicans. J Med Microbiol 40, 416-423.

Rustchenko-Bulgac, E. (1991). Variations of Candida albicans electrophoretic karyotypes. J Bacteriology 1991, 6586-6596.

Schwan, T. G. \& Hinnebush, B. J. (1998). Bloodstream- versus tick-associated variants of a relapsing fever bacterium. Science $\mathbf{2 8 0}$, 1938-1940.

Silverman, M., Zieg, J., Hilmen, M. \& Simon, M. (1979). Phase variation in Salmonella: genetic analysis of a recombinational switch. Proc Natl Acad Sci U S A 76, 391-395.

Sinha, H., Pain, A. \& Johnstone, K. (2000). Analysis of the role of recA in phenotypic switching of Pseudomonas tolaasii. J Bacteriol 182, 6532-6535.

Slutsky, B., Buffo, J. \& Soll, D. R. (1985). High-frequency switching of colony morphology in Candida albicans. Science 230, 666-669.

Slutsky, B., Staebell, M., Anderson, J., Risen, L., Pfaller, M. \& Soll, D. R. (1987). "White-opaque transition": a second high-frequency switching system in Candida albicans. J Bacteriol 169, 189-197.

Soll, D. R. (1992). High-frequency switching in Candida albicans. Clin Microbiol Rev 5, 183-203.

Soll, D. R. (2002). Candida commensalism and virulence: the evolution of phenotypic plasticity. Acta Trop 81, 101-110.

Soll, D., Galask, R., Isley, S., Rao, T., Stone, D., Hicks, J., Schmid, J., Mac, K. \& Hanna, C. (1989). Switching of Candida albicans during successive episodes of recurrent vaginitis. J Clin Microbiol 27, 681-690.

Spitzer, E. D., Spitzer, S. G., Freundlich, L. F. \& Casadevall, A. (1993). Persistence of initial infection in recurrent Cryptococcus neoformans meningitis. Lancet 341, 595-596.

Srikantha, T., Tsai, L., Daniels, K., Klar, A. J. \& Soll, D. R. (2001). The histone deacetylase genes HDA1 and RPD3 play distinct roles in regulation of high-frequency phenotypic switching in Candida albicans. J Bacteriol 183, 4614-4625.

Steenbergen, J. N., Shuman, H. A. \& Casadevall, A. (2001). Cryptococcus neoformans interactions with amoebae suggest an explanation for its virulence and intracellular pathogenic strategy in macrophages. Proc Natl Acad Sci U S A 98, 15245-15250.

Sukroongreung, S., Lim, S., Tantimavanich, S., Eampokalap, B., Carter, D., Nilakul, C., Kulkeratiyut, S. \& Tansuphaswadikul, S. (2001). Phenotypic switching and genetic diversity of Cryptococcus neoformans. J Clin Microbiol 39, 2060-2064.

Swartley, L., Marfin, A., Edupuganti, S., Liu, L., Cieslak, P., Perkins, B. \& Wenger, J. (1997). Capsule switching of Neisseria meningitidis. Proc Natl Acad Sci U S A 94, 271-276.

True, H. L. \& Lindquist, S. L. (2000). A yeast prion provides a mechanism for genetic variation and phenotypic diversity. Nature 407, 477-483.

Vargas, K., Messer, S. A., Pfaller, M., Lockhart, S. R., Stapleton, J. T., Hellstein, J. \& Soll, D. R. (2000). Elevated phenotypic switching and drug resistance of Candida albicans from human immunodeficiency virus-positive individuals prior to first thrush episode. J Clin Microbiol 38, 3595-3607. 